\title{
A SMASH PRODUCT FOR SPECTRA
}

\author{
BY HAROLD M. HASTINGS ${ }^{1}$
}

Communicated by Hyman Bass, January 2, 1973

\begin{abstract}
We shall show that the smash product for pointed CW complexes induces a smash product $\wedge$ on the homotopy category of Adams's stable category with the following properties. $\wedge$ is coherently homotopy unitary $\left(S^{0}\right)$, associative, and commutative, $\wedge$ commutes with suspension up to homotopy, and $\wedge$ satisfies a Kunneth formula.
\end{abstract}

Introduction. Precisely, we shall show that the homotopy category of a technical variant of Adams's stable category [1], a fraction category of $\mathrm{CW}$ prespectra equivalent to that of Boardman [3], [8], admits a symmetric monoidal structure in the sense of [4].

Whitehead's pairings of prespectra [9] and Kan and Whitehead's nonassociative smash product for simplicial spectra [6] were the first attempts at a smash product. Boardman gave the first homotopy associative, commutative, and unitary smash product in his stable category [3]. Adams has recently obtained a similar construction [2].

We acknowledge with thanks many helpful conversations with Professors Alex Heller and John C. Moore. Details and applications will appear elsewhere.

1. The interchange problem. A $S^{k}$-prespectrum $X$ consists of a sequence of pointed spaces $\left\{X_{n} \mid n \geqq 0\right\}$, together with inclusions $X_{n} \wedge S^{k} \rightarrow X_{n+1}$. Consider $S=\left\{S_{n}=S^{n k}\right\}$ as a ring with respect to the smash product of spaces. Then $X$ is a right $S$-module.

Construction of a homotopy associative smash product for $S^{k}$-prespectra requires permutations $\pi$ of $S^{k} \wedge \cdots \wedge S^{k}$. Since $S$ is not strictly commutative, but only graded homotopy commutative, this requires defining suitably canonical maps of degree -1 (for $k$ odd) and homotopies $\pi \simeq \pm$ id.

We avoid sign problems by using $S^{4}$-prespectra and define canonical homotopies $H_{\pi}$ as follows. Make the standard identifications

$S^{4 k} \cong S^{4} \wedge \cdots \wedge S^{4} \cong I^{4} / \partial I^{4} \wedge \cdots \wedge I^{4} / \partial I^{4} \cong I^{4 k} / \partial I^{4 k} \cong D^{4 k} / S^{4 k-1}$.

Then $\pi$ simply permutes factors of $C^{2} \times \cdots \times C^{2}$. Hence $\pi \in S U(2 k)$.

AMS (MOS) subject classifications (1970). Primary 18D45, 55J99; Secondary 55J10, $55 \mathrm{~J} 25$.

Key words and phrases. Symmetric monoidal category, Adams's stable category, Boardman's stable category, special unitary group, Kunneth formula.

${ }^{1}$ Partially supported by a NSF institutional grant. 
Since $S U(2 k)$ is path connected and simply connected, there is a unique homotopy class of paths $\left[\Gamma_{\pi}\right]$ in $S U(2 k)$ with $\Gamma_{\pi}(0)=\pi, \Gamma_{\pi}(1)=e$. Define homotopies $H_{\pi}: S^{4 k} \wedge I^{*} \rightarrow S^{4 k}$ by $H_{\pi}(s, t)=\Gamma_{\pi}(t)(s)$. Then $\left[H_{\pi}\right]$ is the required homotopy class (rel the endpoints) of canonical homotopies.

THEOREM 1. Let $\pi$ and $\pi^{\prime}$ be permutations of $S^{4 k}$. Let $H_{\pi}$ and $H_{\pi^{\prime}}$ be canonical homotopies. Then $H_{\pi^{\prime}}\left(H_{\pi}(s, t), t\right): S^{4 k} \wedge I^{*} \rightarrow S^{4 k}$ is a canonical homotopy for $\pi^{\prime} \pi$.

Adams solves the interchange problem directly for $S^{1}$-prespectra by an argument involving special classes of paths in $S O$.

2. The Adams completion. Give $S^{4}$ the $\mathrm{CW}$ structure with one 4-cell and one 0 -cell. Give $[0, n]$ the $\mathrm{CW}$ structure with 0 -cells $0,1, \ldots, n$.

DEFINITION 2. A prespectrum consists of a sequence of pointed $\mathrm{CW}$ complexes $\left\{X_{n} \mid n \geqq 0\right\}$, together with inclusions as subcomplexes $X_{n} \wedge S^{4} \rightarrow X_{n+1}$. A strict (weak) map of prespectra $f: X \rightarrow Y$ consists of a sequence of continuous maps $\left\{f_{n}: X_{n} \rightarrow Y_{n}\right\}$ such that $f_{n+1}=f_{n} \wedge S^{4}$ (resp. $f_{n+1} \simeq f_{n} \wedge S^{4}$ ) on $X_{n} \wedge S^{4}$. Ps is the category of prespectra and strict maps.

Definition 3 (Adams). A subspectrum $X^{\prime} \subset X$ is cofinal if for each cell $\sigma$ of $X$ a sufficiently high suspension of $\sigma$ is in $X^{\prime}$.

DEFinition 4 (ADAMS). Ad is the category of prespectra in which maps from $X$ to $Y$ are diagrams $X \supset X^{\prime} \rightarrow Y$ in Ps with $X^{\prime}$ cofinal in $X$.

Formally, Ad is the right fraction category [5] of Ps in which cofinal inclusions are invertible. Morphisms $X \supset X^{\prime} \stackrel{f}{\rightarrow} Y$ and $X \supset X^{\prime \prime} \stackrel{g}{\rightarrow} Y$ are equal if $f=g$ on $X^{\prime} \cap X^{\prime \prime}$.

DEFINITION 5. Let $X$ be a prespectrum. Given a monotone unbounded sequence of nonnegative integers $\left\{j_{n} \mid n \geqq 0, j_{n} \leqq n\right\}$, define a prespectrum $D X$ by $(D X)_{n}=X_{j_{n}} \wedge S^{4\left(n-j_{n}\right)}$, with inclusions $(D X)_{n} \wedge S^{4} \rightarrow$ $(D X)_{n+1}$ defined so that $D X \subset X$.

Then $D$ extends to a functor (destabilization) on Ps, and there are natural cofinal inclusions $D X \subset X$.

There are smash products $\wedge: \mathrm{Ps}, \mathrm{CW} \rightarrow \mathrm{Ps}$, and $\wedge: \mathrm{Ad}, \mathrm{CW} \rightarrow \mathrm{Ad}$; these are defined degreewise.

Definition 6. Maps $f, g: X \rightrightarrows Y$ in Ps (Ad) are homotopic if there is a map $H: X \wedge I^{*} \rightarrow Y$ in Ps (resp. Ad) with $H\left|X \wedge 0^{*}=f, H\right| X \wedge 1^{*}=$ g.

Homotopy has the usual properties. Denote the resulting homotopy categories $H t(\mathrm{Ps}), H t(\mathrm{Ad})$.

3. Smash products. We shall define a family of smash products $\wedge$ on $H t(\mathrm{Ad})$. 
DEFINITION 7. Let $X$ and $Y$ be prespectra. Given a sequence of pairs of nonnegative integers $\left\{\left(i_{n}, j_{n}\right) \mid n \geqq 0, i_{n}+j_{n}=n\right.$, and $\left\{i_{n}\right\}$ and $\left\{j_{n}\right\}$ are monotone unbounded sequences $\}$, let $X \wedge Y$ be the prespectrum with $(X \wedge Y)_{n}=X_{i_{n}} \wedge Y_{j_{n}}$; the required inclusions are induced from $X$ and $Y$.

Then $\wedge$ extends successively to bifunctors on Ps, Ad (since the smash product of cofinal inclusions is cofinal) and $H t(\mathrm{Ad})$.

\section{Uniqueness and the symmetric monoidal structure.}

Definition 8. Let $X$ be a prespectrum. A permutation $\Pi$ of $D X$ consists of a sequence of maps $\Pi_{n}=X_{j n} \wedge \pi_{n}:(D X)_{n}=X_{j n} \wedge S^{4} \wedge \cdots \wedge S^{4} \rightarrow$ $D X_{n}$, where each $\pi_{n}$ is a permutation of $S^{4} \wedge \cdots \wedge S^{4}$. If $g=\left\{g_{n}:(D X)_{n} \rightarrow\right.$ $Y_{n} \mid g_{n+1}$ extends $g_{n} \wedge S^{4}$ up to permutation $\}$, call $g$ a permutation map.

Proposition 9. Permutation maps are weak maps, where the required homotopies $H_{n}:(D X)_{n} \wedge S^{4} \wedge I^{*} \rightarrow Y_{n+1}$ are induced by canonical homotopies $(\$ 1)$.

Also, permutation maps may be destabilized and are closed under the following composition: $f: D X \rightarrow Y$ and $g: D^{\prime} Y \rightarrow Z$ yield $g D^{\prime}(f): D^{\prime} D X \rightarrow$ $D^{\prime} Y \rightarrow Z$. Two permutation maps $f, g: D X \rightrightarrows Y$ differ by a permutation if for some permutation $\Pi$ of $D X, g=f \Pi$.

TheOREM 10. Any two smash products $\wedge$ and $\wedge^{\prime}$ on $H t(\mathrm{Ad})$ are naturally isomorphic.

Proof. There are natural destabilizations and permutation classes of permutation maps $D(X \wedge Y) \rightarrow X \wedge^{\prime} Y, D^{\prime}\left(X \wedge^{\prime} Y\right) \rightarrow X \wedge Y$. The composites $D^{\prime} D(X \wedge Y) \rightarrow X \wedge Y$ and $D D^{\prime}\left(X \wedge^{\prime} Y\right) \rightarrow X \wedge^{\prime} Y$ differ from the respective (cofinal) inclusions by permutations. Thus it suffices to prove the following.

LEMMA 11. A permutation commutative diagram of permutation maps $D X \rightarrow Y$ induces a commutative diagram in $\mathrm{Ht}(\mathrm{Ad})$.

We shall sketch a proof in $\$ 6$.

THEOREM 12. There are natural maps in Ht(Ad),

$$
\begin{array}{ll}
X \rightarrow X \wedge S^{0} \rightarrow X & \text { (unit), } \\
a:(X \wedge Y) \wedge Z \rightarrow X \wedge(Y \wedge Z) & \text { (associativity), } \\
c: X \wedge Y \rightarrow Y \wedge X & \text { (commutativity), }
\end{array}
$$

which form a symmetric monoidal category.

Proof. There are natural destabilizations and permutation classes of permutation maps $D X \rightarrow X \wedge S^{0} \rightarrow X$,

$$
a^{\prime}: D(X \wedge Y) \wedge Z \rightarrow X \wedge(Y \wedge Z)
$$


and

$$
c^{\prime}: D(X \wedge Y) \rightarrow Y \wedge X
$$

By Lemma 11, it suffices to obtain the coherency diagrams [4] as permutation-commutative diagrams of permutation maps involving destabilizations. For example, the diagram for coherency of associativity is

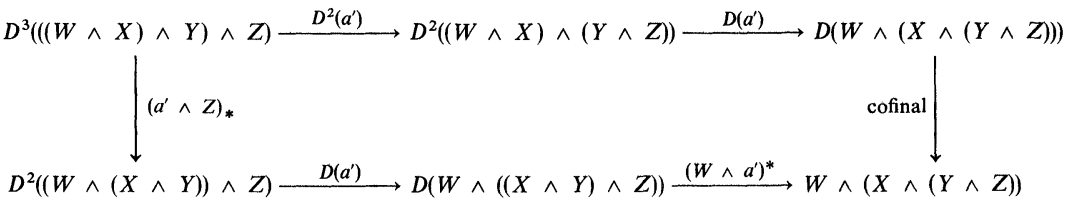

where $D$ is used generically, and $\left(a^{\prime} \wedge Z\right)_{*}$ and $\left(W \wedge a^{\prime}\right)_{*}$ are representatives of permutation classes of permutation maps induced by $a^{\prime}$.

These diagrams are readily obtained.

5. Telescopes. We sketch the main properties of the following telescope construction.

Definition 13. For a prespectrum $X$, let Tel $X$ be the prespectrum with $(\text { Tel } X)_{n}=\bigcup_{j=0}^{n}\left(X_{j} \wedge S^{4(n-j)} \wedge[j, n]^{*}\right)$, the iterated mapping cylinder of $X_{0} \wedge S^{4 n} \rightarrow \cdots \rightarrow X_{n-1} \wedge S^{4} \rightarrow X_{n}$, together with the induced inclusions.

Then Tel may be extended to a functor, and there are natural projections $p_{X}:$ Tel $X \rightarrow X$.

Proposition 14. $p_{X}$ admits a homotopy inverse $s_{X}$.

Proof. To define $s_{X}$, show that Tel $X$ and $X$ are strong deformation retracts of the prespectrum $Y$ with $Y_{n}=X_{n} \wedge[0, n]^{*}$ such that $p_{X}$ is the composite Tel $X \rightarrow Y \rightarrow X$.

Proposition 15. A weak map $f: X \rightarrow Y$, together with a family of homotopies for $f,\left\{H_{n}: X_{n} \wedge S^{4} \wedge I^{*} \rightarrow Y_{n+1}\right.$ from $f_{n} \wedge S^{4}$ to $\left.f_{n+1} \mid X_{n} \wedge S^{4}\right\}$, induces strict maps $\phi:$ Tel $X \rightarrow \operatorname{Tel} Y$ and $F: X \rightarrow Y$.

Proof. Let $\phi_{0}=f_{0}$, and for $n \geqq 0$, define $\phi_{n+1}$ by

$$
\begin{aligned}
\phi_{n+1}(x, t) & =\left(\phi_{n} \wedge S^{4}\right)(x, t) & & \text { for } t \leqq n \\
& =\left(\left(f_{n} \wedge S^{4}\right)(x), 2 t-n\right) & & \text { for } n \leqq t \leqq n+\frac{1}{2} \\
& =\left(H_{n}(x, 2 t-2 n-1), n+1\right) & & \text { for } n+\frac{1}{2} \leqq t \leqq n+1 .
\end{aligned}
$$

Also, let $F=p_{Y} \phi s_{X}$.

Proposition 16. Let $\left\{H_{n}\right\}$ and $\left\{H_{n}^{\prime}\right\}$ be homotopies for a weak map $f: X \rightarrow Y$, and assume that $H_{n} \simeq H_{n}^{\prime}$ rel the endpoints for all $n$. Then 
$\left(f,\left\{H_{n}\right\}\right)$ and $\left(f,\left\{H_{n}^{\prime}\right\}\right)$ induce homotopic strict maps Tel $X \rightarrow \operatorname{Tel} Y$ and $X \rightarrow Y$.

Proposition 17. Let $f^{\prime}: X \rightarrow Y$ and $f^{\prime \prime}: Y \rightarrow Z$ be weak maps with homotopies $\left\{H_{n}^{\prime}\right\}$ and $\left\{H_{n}^{\prime \prime}\right\}$, respectively. Then there are composed homotopies $\left\{H_{n}\right\}$ for $f^{\prime \prime} f^{\prime}$ such that for the respective induced strict maps $F^{\prime}: X \rightarrow Y, F^{\prime \prime}: Y \rightarrow Z$, and $F: X \rightarrow Z, F \simeq F^{\prime \prime} F^{\prime}$.

6. Proof of Lemma 11. Given a permutation-commutative square in which each map and composite (see $\$ 4$ ) is a permutation map,

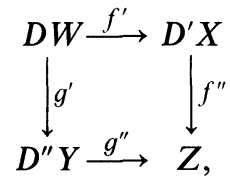

replace each map by a strict map to obtain a commutative square in $H t(\mathrm{Ps})$ (by Theorem 1 , and $\S 5$ ).

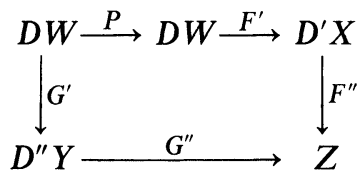

Here $P$ is induced by a permutation $\Pi$ of $D W$. Since, for all $k, S U(2 k)$ is simply connected, we may "pull back" canonical homotopies to obtain a homotopy $P \simeq D W$. This completes the proof, since cofinal inclusions are inverted in $H t(\mathrm{Ad})$.

7. Further properties. $\wedge$ commutes with the suspension

$$
? \wedge S^{1}: H t(\mathrm{Ad}) \rightarrow H t(\mathrm{Ad}),
$$

$\wedge$ satisfies a Kunneth formula for stable integral homology (see [6]), and $\wedge$ is weakly universal for pairings (see [9], [6]). We conjecture the existence of an internal mapping functor adjoint to $\wedge$ using a suitable category of permutation maps and methods of Quillen [7].

ADDED IN PROOF. This follow's from Brown's Theorem by Heller [Trans. Amer. Math. Soc. 147 (1970), 573-602].

\section{REFERENCES}

1. J. F. Adams, Algebraic topology in the last decade, Proc. Sympos. Pure Math., vol. 22, Amer. Math. Soc., Providence, R.I., 1971, pp. 1-23.

2. - Stable homotopy and generalized homology, notes by R. Ming, University of Chicago Math. Lecture Notes, 1971 (mimeographed). 
3. J. M. Boardman, Stable homotopy theory, University of Warwick mimeo, 1965 onwards.

4. S. Eilenberg and G. M. Kelly, Closed categories, Proc. Conf. on Categorical Algebra (La Jolla 1965), Springer, New York, 1966, pp. 421-562. MR 37 \# 1432.

5. P. Gabriel and M. Zisman, Calculus of fractions and homotopy, Ergebnisse der Math. und ihrer Grenzgebiete, Band 35, Springer-Verlag, New York, 1967. MR 35 \# 1019.

6. D. M. Kan and G. W. Whitehead, The reduced join of two spectra, Topology 3 (1965), suppl. 2, 239-261. MR 31 \#2720.

7. Daniel Quillen, Homotopical algebra, Lecture Notes in Math., vol. 43, SpringerVerlag, Berlin, 1967. MR 36 \#6480.

8. R. Vogt, Boardman's stable homotopy category, Aarhus Universitet Lecture Notes, no. 21, 1971 (mimeographed).

9. G. W. Whitehead, Generalized homology theories, Trans. Amer. Math. Soc. 102 (1962), 227-283. MR 25 \#573.

Department of Mathematics, Hofstra University, Hempstead, New York 11550 